\title{
Clinical correlation of biopsy results in patients with temporal arteritis
}

\author{
Volkan Yuksel ${ }^{1 *}$, Orkut Guclu ${ }^{2}$, Ebru Tastekin ${ }^{3}$, Umit Halici ${ }^{4}$, Serhat Huseyin ${ }^{2}$, Volkan Inal ${ }^{5}$, Suat Canbaz ${ }^{6}$ \\ ${ }^{1}$ Associate Professor of Cardiovascular Surgery, Trakya University, Edirne, Turkey

Study conducted at Trakya University Faculty of Medicine, Edirne, Turkey

Article received: 4/5/2017 Accepted for publication: 5/7/2017

*Correspondence:

Trakya University Hospitals Cardiovascular Surgery Department Edirne - Turkey

Postal code: 22100 drvyuksel@yahoo.com

http://dx.doi.org/10.1590/1806-9282.63.11.953

\section{SUMmARY}

Objective: Temporal arteritis is systemic vasculitis of medium and large sized vessels. The lowest incidence rates were reported in Turkey, Japan and Israel. We aimed to investigate the results of patients with biopsy-proven temporal arteritis and those classified according to the American College of Rheumatology criteria from a low-incidence region for temporal arteritis. The results of our study are noteworthy, since there is limited data on pathologic diagnosis of temporal arteritis in Turkey.

Method: We studied the medical records, laboratory findings such as erythrocyte sedimentation rate and C-reactive protein levels, biopsy results, and postoperative complications of all the patients operated for temporal artery biopsy at our clinic. We used the computerized laboratory registry that keeps all records of 42 consecutive temporal artery biopsy results from January 2011 to December 2016. Results: The mean age was $66 \pm 12.5$ years. The most common manifestations on admission were temporal headache, optic neuritis and jaw claudication, respectively. Temporal artery biopsy results confirmed tempoal arteritis in eight out of 42 (19\%) patients. There was no statistically significant difference between biopsy-positive and biopsy-negative groups in terms of sex, age, erythrocyte sedimentation rate, C-reactive protein and biopsy length.

Conclusion: We were not able to find a correlation between the analysis of biopsy results and clinical evaluation of patients with temporal arteritis. We suggest that diagnosis of temporal arteritis depends on clinical suspicion. Laboratory examination results may not be helpful in accurate diagnosis of tempoal arteritis.

Keywords: giant cell arteritis, biopsy, C reactive protein, blood sedimentation.

\section{INTRODUCTION}

Temporal arteritis (TA) is defined as the systemic vasculitis of medium- and large-sized vessels and affects mainly women above 50 years of age. ${ }^{1}$ It is an arterial inflammatory disease mostly affecting temporal arteries and is histologically characterized by arterial infiltration of giant cells. ${ }^{2}$ Patients can present with visual disturbances, jaw claudication, scalp tenderness, headache and myalgia. Clinical evaluation and suspicion are important in diagnosis. Other than clinical evaluation and laboratory assessment, duplex ultrasonography, magnetic resonance imaging and positron emission tomography can also be used for evaluation. Temporal artery biopsy (TAB) is a low-sensitivity but gold standard method in the diagnosis of TA.

The highest incidence rates of TA are reported in Scandinavian countries and northern United States. The lowest incidence rates were reported from Turkey, Japan, Israel and among native Alaskans. ${ }^{3,4}$ A lower frequency of TA in our center in northwestern Turkey was shown compared to European data. ${ }^{5}$

In this study, we aimed to investigate and discuss the clinical correlation of patients with temporal artery biopsy-proven TA and those classified according to the Amer- 
ican College of Rheumatology (ACR) criteria from a low incidence region for TA. ${ }^{6}$ The results of our study are noteworthy, since there is limited data on pathologic diagnosis of TA in that region.

\section{Method}

We performed a retrospective study to evaluate Turkish patients who fulfilled the ACR criteria for TA and were under regular follow-up in our tertiary university hospital. ${ }^{6}$ Detailed clinical history and physical examination were routinely performed in all patients. We studied the medical records, laboratory findings such as erythrocyte sedimentation rate (ESR) and C-reactive protein levels (CRP), biopsy results and postoperative complications of all the patients operated for temporal artery biopsy at our clinic. We used the computerized laboratory registry that keeps all records of 42 consecutive $\mathrm{TAB}$ results from January 2011 to December 2016.

All biopsies were performed using samples taken from the symptomatic side and based on high clinical suspicion bilaterally. Biopsy was performed from superficial temporal artery segment, which is a branch of external carotid artery. Location of the artery was determined prior to operation. Operations were performed under local anesthesia. A careful incision was made to explore the frontal branch of the superficial temporal artery at the temples and maximum care was taken in order not to injure the temporal branch of the facial nerve. The superficial temporal artery must be ligated proximally and distally after deciding the necessary length of biopsy. The skin incision is closed subcutaneously after controlling the bleeding of the side branches. Inflammation (red arrow), especially affecting the intima and medial layers of the arterial wall and causing degeneration of internal elastic lamina with characteristic multinuclear giant cells (blue arrow), lymphocytes, neutrophils and histiocytes, is helpful in pathologic diagnosis for TA (Figure 1). We collected and compared the results of patients with biopsy-proven TA and those classified according to ACR criteria. Ethical approval was obtained from the local ethics committee for this retrospective study.

\section{Statistical analysis}

Statistical Package for the Social Sciences (SPSS) software version 22.0 (SPSS Inc., Chicago, Illinois, USA) and GraphPad prisma 6 (GraphPad software, La Jolla, CA, USA) were used to carry out the statistical analysis. Categorical data are presented as numbers and percentages, while numerical data are presented as means and standard deviations. Differences between patients were analyzed using the Mann-Whitney $U$ test for continuous variables and Chi-square test for categorical variables. $\mathrm{p}<0.05$ was considered significant.

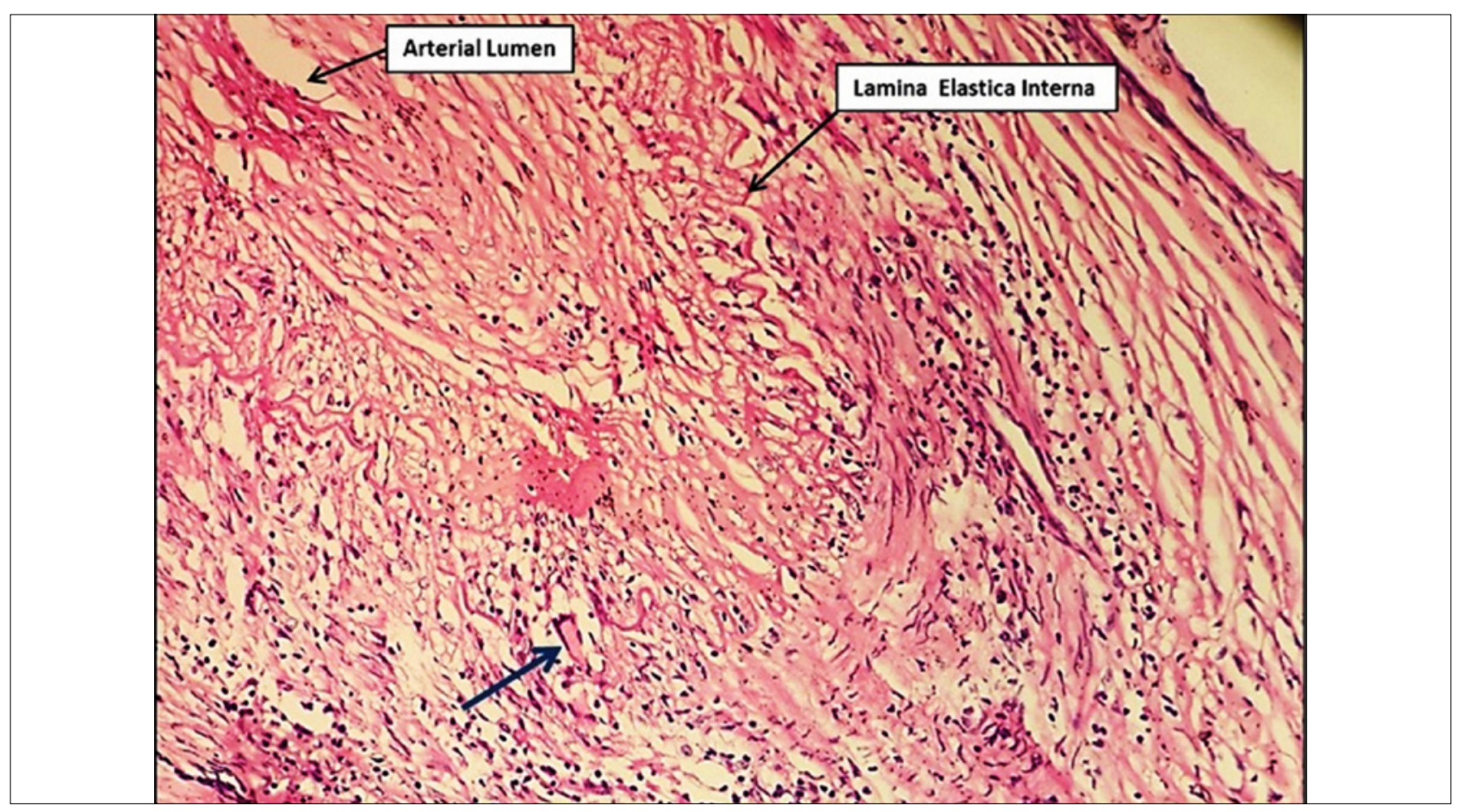

FIGURE 1 Histologic specimen of temporal arteritis. 


\section{RESULTS}

The results and medical records of 42 patients were studied. There were 22 male and 20 female patients and the mean age was $66 \pm 12.5$ years $(25-85)$. The most common manifestations on admission were temporal headache, optic neuritis and jaw claudication, respectively. The clinical symptoms of the patients in the study group are summarized in Table 1.

\section{TABLE 1 Clinical symptoms of patients with} temporal arteritis.

\begin{tabular}{ll} 
Symptom & $\mathbf{n}(\%)$ \\
\hline Temporal headache & $37(88.1)$ \\
\hline Optic neuritis & $28(66.7)$ \\
\hline Jaw claudication & $26(61.9)$ \\
\hline Weight loss & $18(42.9)$ \\
\hline Visual alteration & $15(35.7)$ \\
\hline Polymyalgia rheumatica & $12(28.6)$ \\
\hline Fever & $10(23.8)$
\end{tabular}

TAB was performed from the left temporal artery in 17 patients and from the right temporal artery in 23 patients and bilateral in two patients. The mean biopsy length was $14.2 \pm 4.4 \mathrm{~mm}(5-20 \mathrm{~mm})$. TAB results confirmed TA in eight (19\%) patients. Of the eight biopsy-proven patients, four (50\%) were male and four (50\%), female. $\mathrm{TAB}$ was diagnosed in two cases that were operated bilaterally. No complications were observed postoperatively.

We compared the results of patients with biopsyproven TA and those classified according to ACR criteria in terms of sex, age, ESR, CRP and biopsy length. There was no statistically significant difference between TABpositive and TAB-negative groups. The details are summarized in Table 2.

\section{Discussion}

$\mathrm{TA}$ is the most common systemic vasculitis in patients over 50 years of age. The presence of three out of five criteria defined by the American College of Rheumatology may help physicians to establish the diagnosis with 94\% sensitivity and $91 \%$ specificity: age over 50 years at time of onset, new-onset localized headache, tenderness or decreased pulse of the temporal artery, ESR over 50 $\mathrm{mm} / \mathrm{h}$, and positive TAB. ${ }^{6}$ Treatment is mainly based on high doses of steroids after clinical diagnosis, especially in patients with visual complaints. The prevalence of TA in our region was found to be lower than that in western populations, including southern Europe. ${ }^{5}$

\begin{tabular}{|c|c|c|c|}
\hline & TAB-negative & TAB-positive & $\mathbf{p}$ \\
\hline Female / Male & $16 / 18$ & $4 / 4$ & 1.000 \\
\hline Age (Mean,range) & $66.4 \pm 11.2$ & $64.1 \pm 18.1$ & 0.847 \\
\hline $\mathrm{ESR}(\mathrm{mm} / \mathrm{hr})$ & $54.3 \pm 31.4$ & $55 \pm 36.2$ & 0.949 \\
\hline CRP (mg/dL) & $1.68 \pm 2.82$ & $4.29 \pm 4.78$ & 0.461 \\
\hline Biopsy length (mm) & $14.3 \pm 4.6$ & $14.0 \pm 3.7$ & 0.935 \\
\hline
\end{tabular}

ACR: American College of Rheumatology; CRP: C-reactive protein levels; ESR: erythrocyte sedimentation rate; $\mathrm{TAB}$ : temporal artery biopsy.

Although TAB is the gold standard method for diagnosis of TA, biopsy length, presence of skip lesions and steroid treatment prior to biopsy may cause false negative results. ${ }^{7}$ Most of the patients have a negative biopsy and this biopsy result does not usually change the management of the patient. For this reason, the role of TAB is not accurately defined. Biopsy results give an idea of disease prognosis and avoid unnecessary treatment. ${ }^{8-10}$

The most common symptom in patients with TA is headache, which is defined as severe, acute and different from previous ones. ${ }^{11}$ Visual loss, jaw claudication, tenderness in temporal region and decreased or lack of pulsation of temporal artery may accompany. In our study group, temporal headache, optic neuritis and jaw claudication were the commonest complaints.

TA is characterized histologically by inflammatory infiltration of the arterial wall by lymphocytes, macrophages and giant cells. There is an accumulation of histiocytes, epithelioid cells and giant cells and dissolution of the elastic lamina. ${ }^{12}$ The important point is that pathological changes are usually segmental and some patients may have a false negative biopsy, and therefore biopsies can be performed in highly suspected selected cases. ${ }^{13}$ Treatment is mainly based on steroids and usually started after confirmation of the diagnosis, and should not be delayed while waiting for $\mathrm{TAB}$ results.

Biopsy was performed bilaterally in two patients with bi-temporal headache on admission and both were diagnosed as TA. An additional contralateral biopsy may be beneficial in terms of high clinical suspicion of TA. ${ }^{14}$ The mean length of TAB specimen after fixation was $14.2 \pm 4.4 \mathrm{~mm}$ in the study group. Mahr et al. also recommend a fixed TAB length of at least $5 \mathrm{~mm}$, sufficient to make a histological diagnosis of TA..$^{15}$ Another factor affecting positive biopsy results may be that our region presents one of the 
lowest incidences of TA. ${ }^{5} \mathrm{~A}$ positive biopsy result is proof of TA, but a negative result does not rule it out.

TAB results confirmed TA in 19\% of our study group. Mahr et al. ${ }^{15}$ reported a $15 \%$ histological evidence of TA in 1,520 patients. Kermani et al. ${ }^{16}$ also found $22 \%$ accuracy for pathological diagnosis in 1,106 patients. However, Suelves et al. ${ }^{17}$ presented $68.4 \%$ positive biopsy results in their study. TA is known to affect women more than men. But in our study group, the ratio was found to be equal. Pamuk et al. ${ }^{5}$ also reported a male to female ratio of 9:10 from the same region, which can be interpreted as nearly equal. Saedon et al ${ }^{18}$ reported a male-female ratio of 3:2 in a group of 153 patients undergoing TAB. The proportion of the biopsy results may vary in studies from different parts of the world.

We compared the results of patients with biopsyproven TA and those classified according to ACR criteria in terms of sex, age, ESR, CRP and biopsy length, and there was no significant difference between groups. Also Souza et al. ${ }^{19}$ reported no significant difference between groups in terms of age, sex and ESR levels. Due to the postmenopausal onset of TA, sex-based hormonal differences do not play a protective role against autoimmunity anymore.

$\mathrm{TAB}$ procedure is a simple and safe procedure, but some complications such as hematoma, scalp necrosis, wound infection, facial nerve injury and stroke may arise. ${ }^{20,21}$ In our study group, we did not observe any complications postoperatively.

The limitations of our study are the small sample size due to low incidence of the disease in our region and the fact that the results are not representative of the entire population, including only patients from a tertiary hospital.

\section{Conclusion}

In conclusion, we were not able to find a correlation between the analysis of biopsy results and the clinical evaluation of patients with temporal arteritis. We suggest that diagnosis of TA mainly depends on high clinical suspicion. Laboratory examination results may not be helpful for an accurate diagnosis of TA. TAB is still the gold standard technique and can be performed safely. TAB is a simple procedure that can be performed under local anesthesia, and it may help definitive diagnosis of TA with low sensitivity. Results may vary in countries where TA is rare compared to other countries.

\section{ACKNOWLEDGMENTS}

We thank Fatma Nesrin Turan for her support in statistical analysis.

\section{Conflict of InTERest}

The authors declare no conflict of interest.

\section{References}

1. Nordborg E, Nordborg C. Giant cell arteritis: epidemiological clues to its pathogenesis and an update on its treatment. Rheumatology (Oxford). 2003; 42(3):413-21.

2. Alberts M. Temporal arteritis: improving patient evaluation with a new protocol. Perm J. 2013; 17(1):56-62.

3. Borchers AT, Gershwin ME. Giant cell arteritis: a review of classification, pathophysiology, geoepidemiology and treatment. Autoimmun Rev. 2012; 11(6-7):A544-54.

4. Gonzales-Gay MA, Vazquez-Rodriguez TR, Lopez-Diaz MJ, Miranda-Filloy JA, Gonzalez-Juanatey C, Martin J, et al. Epidemiology of giant cell arteritis and polymyalgia rheumatica. Arthritis Rheum. 2009; 61(10):1454-61.

5. Pamuk ON, Dönmez S, Karahan B, Pamuk GE, Cakir N. Giant cell arteritis and polymyalgia rheumatica in northwestern Turkey: clinical features and epidemiological data. Clin Exp Rheumatol. 2009; 27(5):830-3.

6. Hunder GG, Bloch DA, Michel BA, Stevens MB, Arend WP, Calabrese LH, et al. The American College of Rheumatology 1990 criteria for the classification of giant cell arteritis. Arthritis Rheum. 1990; 33(8):1122-8.

7. Bhatti MT, Tabandeh H. Giant cell arteritis: diagnosis and management. Curr Opin Ophthalmol. 2001; 12(6):393-9.

8. Duhaut $\mathrm{P}$, Pinède L, Bornet $\mathrm{H}$, Demolombe-Ragué S, Dumontet $\mathrm{C}$, Ninet J, et al. Biopsy-proven and biopsy negative temporal arteritis: differences in clinical spectrum at the onset of disease. Ann Rheum Dis. 1999; 58(6):335-41.

9. González-Gay MA, Alonso MD, Agüero JJ, Bal M, Fernández-Camblor B, Sánchez-Andrade A. Temporal arteritis in a northwestern area of Spain: study of 57 biopsy proven patients. J Rheumatol. 1992; 19(2):277-80.

10. González-Gay MA, García-Porrúa C. Systemic vasculitis in adults in northwestern Spain, 1988-1997. Clinical and epidemiological aspects. Medicine (Baltimore). 1999; 78(5):292-308.

11. Solomon S, Cappa KG. The headache of temporal arteritis. J Am Geriatr Soc. $1987 ; 35(2): 163-5$

12. Ness T, Bley TA, Schmidt WA, Lamprecht P. The diagnosis and treatment of giant cell arteritis. Dtsch Arztebl Int. 2013; 110(21):376-86.

13. Poller DN, van Wyk Q Jeffrey MJ. The importance of skip lesions in temporal arteritis. J Clin Pathol. 2000; 53(2):137-9.

14. Davies C, Frost B, Eshan O, McLain AD, Shandall A. Temporal artery biopsy... who needs one? Postgrad Med J. 2006; 82(969):476-8.

15. Mahr A, Saba M, Kambouchner M, Polivka M, Baudrimont M, Brochériou $\mathrm{I}$, et al. Temporal artery biopsy for diagnosing giant cell arteritis: the longer, the better? Ann Rheum Dis. 2006; 65(6):826-8.

16. Kermani TA, Schmidt J, Crowson CS, Ytterberg SR, Hunder GG, Matteson EL, et al. Utility of erythrocyte sedimentation rate and C-reactive protein for the diagnosis of giant cell arteritis. Semin Arthritis Rheum. 2012; 41(6):866-71.

17. Suelves AM, España-Gregori E, Aviñó J, Rohrweck S, Díaz-Llopis M. Analysis of factors that determine the diagnostic yield of temporal artery biopsy. Arch Soc Esp Oftalmol. 2013; 88(4):127-9.

18. Saedon H, Saedon M, Goodyear S, Papettas T, Marshall C. Temporal artery biopsy for giant cell arteritis: retrospective audit. JRSM Short Rep. 2012; 3(10):73

19. Souza AW, Okamoto KY, Abrantes F, Schau B, Bacchiega AB, Shinjo SK Giant cell arteritis: a multicenter observational study in Brazil. Clinics (Sao Paulo). 2013; 68(3):317-22.

20. Dummer W, Zillikens D, Schulz A, Bröcker EB, Hamm H. Scalp necrosis in temporal (giant cell) arteritis: implications for the dermatologic surgeon. Clin Exp Dermatol. 1996; 21(2):154-8.

21. Bhatti MT, Goldstein MH. Facial nerve injury following superficial temporal artery biopsy. Dermatol Surg. 2001; 27(1):15-7. 